\title{
Domination on Minimum Diameter Spanning Tree
}

\author{
V.T. Chandrasekaran \\ Department of Mathematics, \\ Jawahar Science College, \\ Neyveli-607803, India. \\ E-mail:vtcvtc14@gmail.com \\ N.Rajasri \\ Department of Mathematics, \\ Vallalar Arts and Science College, \\ Vadalur-607303. India. \\ E-mail: rajasri150583@gmail.com
}

\begin{abstract}
In this paper we present dominating sets, minimum diameter spanning tree for grid graph and complete graph, further discuss the simple connected graphs and above which have a minimum diameter spanning tree such that both have same domination number.
\end{abstract}

Key words: Domination, Diameter, minimum diameter Spanning tree, Complete graph, Grid graph, lollipop graph, web graph, sunlet graph, cocktail graph.

\section{INTRODUCTION}

E.J. Cockayne and S.T. Hedetniemi [1] introduced the concept dominating set. Frank Harary, Robert Z.Norman and Dorwin Cartwright [2] explained an interesting application in voting situations using the concept of domination. C.L. Liu [3] also discussed the application of dominance to communication network, Where a dominating set represents a set of cities which acting as transmitting Stations can transmit messages to ever y city in the network. A subset $\mathrm{S}$ of vertices from $\mathrm{V}$ is called a dominating set for $\mathrm{G}$ if every vertex of $\mathrm{G}$ is either a member of $\mathrm{S}$ or adjacent to a member of $\mathrm{S}$. A dominating set of $\mathrm{G}$ is Called a minimum dominating set if $\mathrm{G}$ has no dominating set of smaller cardinality. The cardinality of minimum dominating set of $G$ is called the dominating number for $\mathrm{G}$ and it is denoted by $\gamma(G)$ Haray, F. (1969) Graph Theory. Addsion Wesley, Reading MA. [4]. Chandrasekaran, V.T. and Rajasri.N (2018) Minimum Diameter Spanning Tree [5]. In this paper, we discuss few simple connected graphs for which the domination numbers of the graph and that of its minimum diameter spanning trees are the same.

The $(\mathrm{m}, \mathrm{n})$ lollipop graph is a graph obtained by joining a complete graph $K_{m}$ to a path $P_{n}$ on $n$ vertices and its denoted by $\mathrm{L}_{\mathrm{m}, \mathrm{n}}[10]$. and the sunlet graph is the graph on vertices obtained by attaching pendent edges to a cycle graph. Sunlet graphs are trivially unit distance, as well as matchsticks graphs [11] and A graph consisting of two rows of paired nodes in which all nodes except the paired ones are connected with straight lines;it is the complement of the ladder graph, and the dual graph of the hypercube .

\section{Some definitions:}

Definition: Let $G=(V, E)$ be a graph. A subset $S$ of $V$ is called dominating set if every vertex in $V-S$ is adjacent to a vertex in $S$. The minimum cardinality of a dominating set in $G$ is called the domination number of $G$ and it is denoted by $\gamma(G)$.

Definition: The spanning tree $T$ of the simple connected graph $G$ is said to be a minimum diameter spanning tree. if there is no other spanning tree $T^{\prime}$ of $G$ such that $d\left(T^{\prime}\right)<d(T)$ The minimum diameter spanning tree is denoted by $M\left(T_{d}\right)$. 


\section{Available online at www.ijrat.org}

Definition: the diameter of a graph is length of the shortest path between the most distance node and it is denoted by $d$.

Theorem 1: If $G$ is a connected graph without pendent vertex. Then $\gamma(G) \leq\left\lceil\frac{n}{d}\right\rceil\left\lceil\frac{d}{3}\right\rceil$ and $\frac{n}{2} \geq d$ where $\mathrm{d}$ - is the diameter of the graph.

Proof

Let $G$ be a any connected graph without pendent vertex, and $d(u, v)$ be a maximum length in $G$, That is $u-v$ path is a minimum diameter.

To prove: $\gamma(G) \leq\left\lceil\frac{n}{d}\right\rceil\left\lceil\frac{d}{3}\right\rceil$, the graph $G$ can have at most $\left\lceil\frac{n}{2}\right\rceil$ unique path of length $d-1$. And the domination number of the path of length $d-1$ can at most be $\left\lceil\frac{d}{3}\right\rceil$. Therefore domination number of the graph can at most be $\left.\left\lceil\frac{n}{d}\right\rceil \frac{d}{3}\right\rceil$.

Therefore, $\gamma(G) \leq\left\lceil\frac{n}{d}\right\rceil\left\lceil\frac{d}{3}\right\rceil$.

Theorem: Let $G$ be a complete graph, then $\gamma(G)=\gamma(T), \gamma\left(G_{1}\right)=\gamma\left(T_{1}\right)$,

$\gamma\left(G_{2}\right)=\gamma\left(T_{2}\right), \ldots . . \gamma\left(G_{n-1}\right)=\gamma\left(T_{n-1}\right)$.

Proof

For every complete graph there exists a minimum diameter spanning tree and also having equal domination. Further, if we reduce the end vertex of the diameter in $\mathrm{G}$ and $\mathrm{T}$ till we reach trivial graph then we get $\gamma(G)=\gamma(T), \gamma\left(G_{1}\right)=\gamma\left(T_{1}\right), \gamma\left(G_{2}\right)=\gamma\left(T_{2}\right), \ldots \ldots \gamma\left(G_{n-1}\right)=\gamma\left(T_{n-1}\right)$.

Sequences of complete graph and minimum diameter spanning tree are denoted as

$$
\begin{array}{cc}
G & T \\
G_{1}=G-v_{1} & T_{1}=T-v_{1} \\
G_{2}=G_{1}-v_{2} & T_{2}=T_{1}-v_{2} \\
G_{3}=G_{2}-v_{3} & T_{3}=T_{2}-v_{3} \\
\ldots \ldots . & \\
\ldots \ldots . . & \ldots \ldots . . . . \\
G_{n-1}=\text { single vertex } & \ldots \ldots . .
\end{array}
$$

Here $v_{1}, v_{2}, v_{3}, \ldots \ldots \ldots . . v_{n-2}$ are the end vertices of the $G_{1}, G_{2}, G_{3 \ldots \ldots \ldots . . .} G_{n-2}$ respectively. 
International Journal of Research in Advent Technology, Vol.7, No.1, January 2019 E-ISSN: 2321-9637

Available online at www.ijrat.org

For example

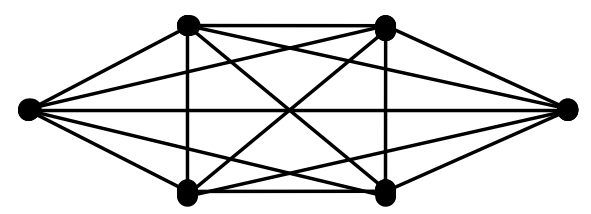

Diameter 1

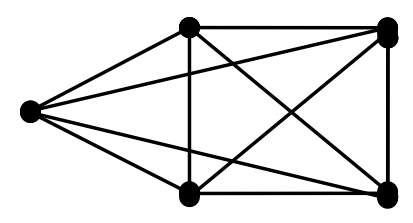

Diameter 1

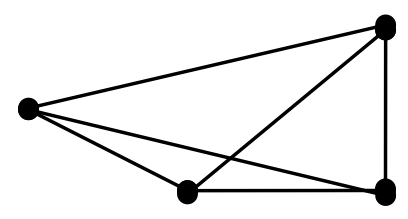

Diameter 1

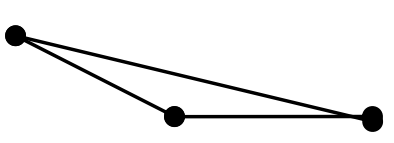

Diameter 1

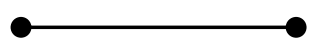

Diameter 1

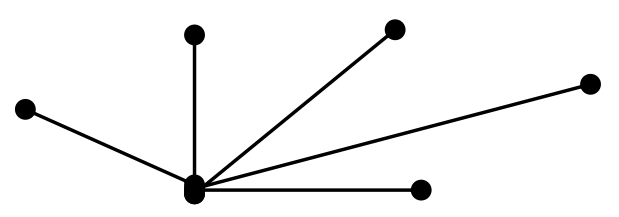

Diameter 2

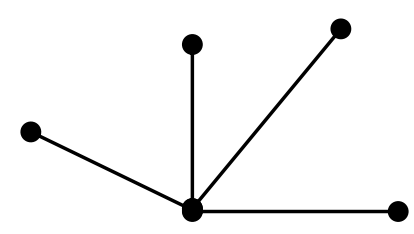

Diameter 2

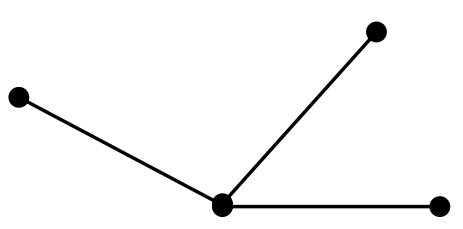

Diameter 2

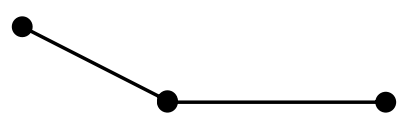

Diameter 2

Diameter 1

\section{Theorem:}

For any graph $G=(V, E)$ is $\gamma(G) \leq \gamma\left(M\left(T_{d}\right)\right)$.

Proof: 


\section{Available online at www.ijrat.org}

For any graph $G$ has $\gamma(G) \leq \gamma\left(M\left(T_{d}\right)\right)$

Since, $M\left(T_{d}\right)$ is the minimum diameter spanning tree with minimum domination number. Clearly $V\left(M\left(T_{d}\right)\right)=V(G)$ and $E(G) E\left(M\left(T_{d}\right)\right)$. As graph $G$ have same or more edge that $M\left(T_{d}\right)$ in graph $G$. A vertex minimum dominating set have more chance to dominate other vertices when compare to dominating set of $M\left(T_{d}\right)$. Therefore, $\gamma\left(M\left(T_{d}\right)\right)$ must be greater than or equal to $\gamma(G)$.

\section{Theorem:}

Let $G=(V, E)$ be a Grid graph. Then $G$ has at least one minimum diameter spanning tree for which $\gamma\left(G_{m, n}\right)=\gamma\left(M\left(T_{d}\right)\right)$.

Proof

The proof is given by the method of induction on the number of rows in $G$.

Clearly,

The result is true for $m=3,4,5$

Than, Assume the result is true for $m=k$, Where $k>2$.

Now, we have to prove that the result is true for $m=k+1$

Let $G$ be a graph with $k+1$ rows,

Let $N$ be the diameter of the graph $G$.

Such that $d(u, v)=N$.

Now consider the graph $H$ obtained by $G-r$.

Then $H$ is a simple finite graph with $\mathrm{k}$ rows

If $H$ is not connected, then there exists a vertex w such that there exist no path between $u \& w$ in $H$.

Therefore in the graph $G$ every path between $u$ and $w$ has a vertex $v$. This means that in the graph $G, d(u, w)>d(u, v)=N$.

i. e. $d(u, w)>N$.

Which is a contradiction, since $N$ is the diameter of $G$. Therefore $r$ is the end row of the graph.

By induction hypothesis $H$ has a minimum diameter spanning tree $T^{1}$ from which $\gamma(H)=\gamma\left(M\left(T_{d}{ }^{1}\right)\right)$.

Now consider the tree $T$ obtained by adding row $\mathrm{r}$ to $T^{1}$ without loss of generality, which is added to nodes where domination is exist, from this graph we get diameter is minimum and then domination also same.

If the domination node does not exist then we have to add any node.

Clearly, $T$ is a spanning tree of $G$ for which $\gamma\left(G_{m, n}\right)=\gamma\left(M\left(T_{d}\right)\right)$.

\section{Result:}

The diameter of $G_{m, n}$ is $m+n-2$ only.

The diameter of $M\left(T_{d}\right)=\left\{\begin{array}{c}m+n-1, m \text { and } n \text { are even } \\ m+n-2, \text { otherwise }\end{array}\right.$ 


\section{Available online at www.ijrat.org}

For some standard graphs $\gamma(G)=\gamma(T)$

*For any cocktail party graph $\gamma(\mathrm{G})=\gamma(\mathrm{T})=2$

*For any sunlet graph $\gamma(\mathrm{G})=\gamma(\mathrm{T})=\left\lfloor\frac{n+1}{2}\right\rfloor$

* For any web graph $\gamma(\mathrm{G})=\gamma(\mathrm{T})=\mathrm{n}$

*For any lollipop graph $\gamma(\mathrm{G})=\gamma(\mathrm{T})$

\section{CONCLUSION}

In this article, we have discussed few graphs for which the domination number is the same that of its minimum diameter spanning tree. Further research can be done in exploring various graphs with the same property. The condition for which a graph does not posses such spanning tree may also be explored.

\section{REFERENCE}

[1] Cockayne, E.J. and Hedetniemi, S.T. (1977) Towards a Theory of Domination in Graphs. Networks, 7, 247261. https://doi.org/10.1002/net.3230070305

[2] Harary, F., Norman, R.Z. and Cartwright, D. (1967) An Introduction to the Theory of Directed Graphs. John Wiley and Sons, New York.

[3] Liu, C.L. (1968) Introduction to Combinatorial Mathematics. McGraw-Hill, New York.

[4] Haray, F. (1969) Graph Theory. Addison Wesley, Reading MA. https://doi.org/10.21236/AD0705364

[5] Chandrasekaran, V.T. and Rajasri, N. (2018) Minimum Diameter Spanning Tree, 203-208. https://www.scirp.org/journal/ajcm

[6] Bray, N. and Weisstein, E.W. (2018) Domination Number. Math World-A Wolfram Web Resource, Champaign.

[7] P.Sharifani ,M.R Hooshmandasl and M.Alambardar Meybodi An explicit construction of optimal dominating and dominating sets in grid.arXiv:1707.0647v3[cs.DM] 2018.

[8] R.Cherifi, S.Gravier and I.Zighem, Bounds on domination number of complete grid graphs, Ars Combin. 60(2001), 307-311.

[9] Samu Alanko, Simon Crevils, Andon Isopoussu, Patric Ostergard and Ville Pettersson ) computing the domination number of grid graphs. The Electronic Journal of Combinatorics 18(2011),\# p141.

[10] Weisstein, Eric W." lollipop graph". math world.

[11] Weisstein, Eric W." sunlet graph". math world. 